\title{
Focal cortical atrophy in multiple sclerosis: Relation to lesion load and disability
}

\author{
Arnaud Charil, ${ }^{\mathrm{a}}$ Alain Dagher, ${ }^{\mathrm{a}, *}$ Jason P. Lerch, ${ }^{\mathrm{a}}$ Alex P. Zijdenbos, ${ }^{\mathrm{a}}$ \\ Keith J. Worsley, ${ }^{\mathrm{a}, \mathrm{b}}$ and Alan C. Evans ${ }^{\mathrm{a}}$ \\ ${ }^{a}$ McConnell Brain Imaging Centre, Montreal Neurological Institute, 3801 University St., Montréal (Québec), Canada H3A 2B4 \\ ${ }^{\mathrm{b}}$ Department of Mathematics and Statistics, McGill University, Montréal, Québec, Canada
}

Received 2 May 2006; revised 15 September 2006; accepted 4 October 2006

\begin{abstract}
Multiple sclerosis (MS) is thought to predominantly affect white matter (WM). Recently, however, loss of cortical gray matter has also been described. Little is known about the cause of cortical atrophy in MS, whether it occurs early in the disease course, and whether it affects all cortical regions equally or if there is a preferential pattern of focal cortical atrophy. An automated method was used to compute the thickness at every vertex of the cortical surface of the brains of $\mathbf{4 2 5}$ early relapsing-remitting MS patients. We correlated cortical thickness with the WM lesion load and the Expanded Disability Status Scale score. Mean cortical thickness correlated with WM lesion load and disability. The correlations of cortical thickness with total lesion load and disability were most significant in cingulate gyrus, insula, and associative cortical regions. Conversely, primary sensory, visual, and motor areas showed a less significant relationship. The highest amount of atrophy per lesion volume or disability scale unit was in the anterior cingulate cortex. This study confirms the relation between cortical atrophy, WM lesion load, and disability in MS, and suggests that cortical atrophy occurs even in MS patients with only mild disability. Most interestingly, we show a specific regional pattern of focal atrophy in MS that is distinctively different from the one in normal aging. The predilection of the atrophic process for areas that are heavily interconnected with other brain regions suggests that interruption of WM tracts by MS plaques contributes, at least in part, to the development of cortical atrophy.
\end{abstract}

(C) 2006 Elsevier Inc. All rights reserved.

\section{Introduction}

Multiple sclerosis (MS) is traditionally described as a multifocal inflammatory demyelinating disease primarily affecting the white matter (WM) of the central nervous system with relative preservation of axons and cell bodies. However, more recent studies have demonstrated the presence of gray matter (GM) lesions (Peterson et al., 2001; Geurts et al., 2005) and brain atrophy

\footnotetext{
* Corresponding author. Fax: +1 5143988948. E-mail address: alain@bic.mni.mcgill.ca (A. Dagher).

Available online on ScienceDirect (www.sciencedirect.com).
}

(Chard et al., 2002; Anderson et al., 2006). Total brain atrophy can be viewed as a surrogate marker of the destructive pathological processes taking place in MS and can be observed with magnetic resonance imaging (MRI) by measuring the brain parenchymal fraction, a normalized measure that represents the sum of both gray matter (GM) and WM atrophy (Chard et al., 2002). In addition to establishing global brain atrophy as a pathological feature of the disease, studies have focused separately on the atrophy of cortical GM by measuring decreases in normalized cortical volumes (De Stefano et al., 2003), by means of an ordinal visual scale (Bakshi et al., 2001), or by directly measuring cortical thickness in vivo (Sailer et al., 2003; Chen et al., 2004). MS-related cerebral atrophy appears to be clinically relevant as it correlates with measures of both disability (Bakshi et al., 2001; De Stefano et al., 2003) and disease progression (Hardmeier et al., 2003; Kalkers et al., 2002).

To date, little is known about the mechanisms involved in cortical atrophy in MS, and whether it is a diffuse process affecting all cortical regions equally or if there is a preferential pattern of focal cortical atrophy. Studies designed to investigate focal changes in cortical thickness in vivo, and their relation to clinical parameters in large groups of MS patients are lacking, possibly because of the technical challenges involved in accurately measuring the thickness of the cortex across the entire brain with its complex folding patterns.

In the present study, an automated method (Lerch et al., 2005) was used to compute the thickness at every vertex of the cortical surface of the brains of 425 relapsing-remitting MS patients. Cortical thickness is one dimension of the cortical volume, the other being surface area. One of the advantages of using cortical thickness as a measure is that it makes a statistical parametric mapping analysis possible and no longer requires the specification of regions of interest.

The novelty of the present study is to investigate cortical changes in a large group of MS patients by measuring the thickness throughout the entire cortex, thus offering a direct quantitative index of cortical atrophy that is more meaningful than GM density or concentration as used in voxel-based morphometry (Ashburner and Friston, 2000). 
Cortical atrophy as defined here refers to a loss of GM that results in thinning of the cortex. The goal of this study was to investigate the relation between overall as well as focal cortical atrophy and both the total WM lesion load (TWMLL) and disability in a large group of relapsing-remitting MS patients.

\section{Patients and methods}

We studied 425 patients with clinically definite relapsingremitting MS (Poser et al., 1983) (228 males; mean age =39 years; $\mathrm{SD}=6$; range $=21-48$ years) who had undergone yearly MRI acquisition and clinical evaluation for 2 years as part of a phase III clinical trial. The study, designed to study the effects of an oral formulation of bovine myelin (Myloral ${ }^{\mathrm{TM}}$, Autoimmune Inc., Lexington MA) (Weiner, 1997; Charil et al., 2003), revealed no differences in frequency of relapses or progression of disability between the treated and placebo groups (Eastwood and Reingold, 1997). All patients had been relapse-free for at least 28 days prior to entering the study. Exclusion criteria for this study included treatment with corticosteroids within the preceding 28 days, treatment with any experimental immunomodulating drug or interferon within the preceding 60 days, and treatment with cyclophosphamide, azathioprine, methotrexate, or cyclosporine within the preceding two years. Patients had tested negative for the presence of human immunodeficiency virus or human T-lymphotropic virus and did not suffer from other serious neurological diseases. This double-blind, placebo controlled, multi-center study was approved by the Institutional Review Boards at the 14 participating centers (four in Canada and ten in the U.S., listed in the acknowledgments) and written informed consent was obtained from all patients.

\section{Disability scores}

All patients were assessed using Kurtzke's expanded disability status scale (EDSS) (Kurtzke, 1983). The scale is weighted towards ambulatory deficits, has a 0.5 step size and ranges from normal $(\mathrm{EDSS}=0)$ to death due to $\mathrm{MS}(\mathrm{EDSS}=10)$.

\section{MRI protocol}

MRI data obtained at 24 months after entry were used because disability and disease burden were higher than at the beginning of the trial. The MRI scans were performed on one of three $1.5 \mathrm{~T}$ cameras at the 14 participating sites (GE Signa, Philips ACS-II, or Siemens SP 4000) using a standard head coil. The acquisition protocol involved a 3-D, T1-weighted gradient echo sequence (TR/ $\mathrm{TE}=35 / 11 \mathrm{~ms}$, flip angle $=45^{\circ}, 60 \times 3 \mathrm{~mm}$ contiguous slices, $256 \times 192$ matrix, $1 \mathrm{NEX}$ ) and a 2D, T2/PD-weighted multi-slice spin-echo sequence $(\mathrm{TR} / \mathrm{TE} 1 / \mathrm{TE} 2=3000 / 30 / 80 \mathrm{~ms}, 54 \times 3 \mathrm{~mm}$ contiguous slices, $256 \times 192$ matrix, $0.5 \mathrm{NEX}$ ) scan. The organizing center (Montreal Neurological Institute) ensured standardization of acquisition procedures prior to the study and quality control of the imaging component throughout. Data were reconstructed to generate a three-dimensional MRI volume for each patient and for each acquisition.

\section{Automated lesion detection}

We used INSECT (Intensity Normalized Stereotaxic Environment for Classification of Tissues), an automatic image-processing pipeline developed at the Montreal Neurological Institute (Zijdenbos et al., 2002). The first step is to transform the native MRIs into a standardized stereotaxic coordinate space based on the Talairach atlas (Talairach and Tournoux, 1988). This procedure uses an automatic, multi-scale feature-matching algorithm (Collins et al., 1994) that performs a 9-parameter linear transformation to match each brain to a template brain. The images were corrected for nonuniformity artifacts (Sled et al., 1998). Subsequently, the registered and corrected volumes were segmented into WM, GM, cerebrospinal fluid, background, and MS lesions using an advanced neural network classifier. A total of six features were used as input to the classifier: three MRI modalities (T1-, T2-, and PD-weighted), and three spatial tissue probability maps (white matter (WM), gray matter (GM), and cerebro-spinal fluid (CSF)) to confine the MS lesion search to plausible white matter areas (Zijdenbos et al., 2002; Kamber et al., 1995). This model-based segmentation has limitations for the detection of lesions occurring in probable gray matter areas, lesions having the same double-echo signature as gray matter, and lesions occurring very near the cortex. These lesion measurements have been shown to be statistically indistinguishable from repeated measurements obtained from trained individuals (Zijdenbos et al., 2002). The linearly transformed MRI images were used for this purpose; therefore, the lesion volume measured here represents relative rather than absolute lesion load. Fig. 1 shows the lesion probability map derived from the 425 individual lesion maps, illustrating for each voxel the likelihood of finding a lesion in this population.

\section{Cortical thickness measurement}

The WM and GM surfaces were fitted using deformable models (Kim et al., 2005), resulting in two surfaces with 81920 polygons each. WM lesion voxels were masked out prior to the fitting of the WM surface to prevent errors due to WM lesions located close to the cortex. This was done by re-labeling lesion voxels as WM voxels for the purpose of fitting the cortical surface.

The surface deformation algorithm (Kabani et al., 2001; Kim et al., 2005) first fits the WM surface, and then expands outward to find the GM/cerebro-spinal fluid intersection. One particularity of this procedure is that each vertex of the WM surface is closely linked to its GM surface counterpart. Cortical thickness can thus be defined as the distance between these linked vertices. The precision of this algorithm, its optimal performance parameters, and the sensitivity of the method to subtle, focal changes in cortical thickness were examined by Lerch and Evans (2005) using a simulated population study and single subject reproducibility metrics. This was shown to be a reliable method, reaching a sensitivity (probability of a true-positive) of 0.93 . That study showed that, given two groups of 25 subjects, a $0.6-\mathrm{mm}(15 \%)$ difference in thickness could be recovered. This algorithm has also been validated by comparison to manual measurements (Kabani et al., 2001). In order to improve sensitivity, each subject's cortical thickness map was blurred using a $20 \mathrm{~mm}$, surface-based blurring kernel (Chung et al., 2003). This diffusion smoothing method follows the curvature of the cortical surface respecting anatomical boundaries, unlike the volumetric blurring typically used in voxel-based morphometry. Twenty millimeters was chosen as the blurring kernel size to provide an optimal balance between sensitivity and precision (Lerch and Evans, 2005). The relevant parts of the processing pipeline are schematically shown in Fig. 2. 


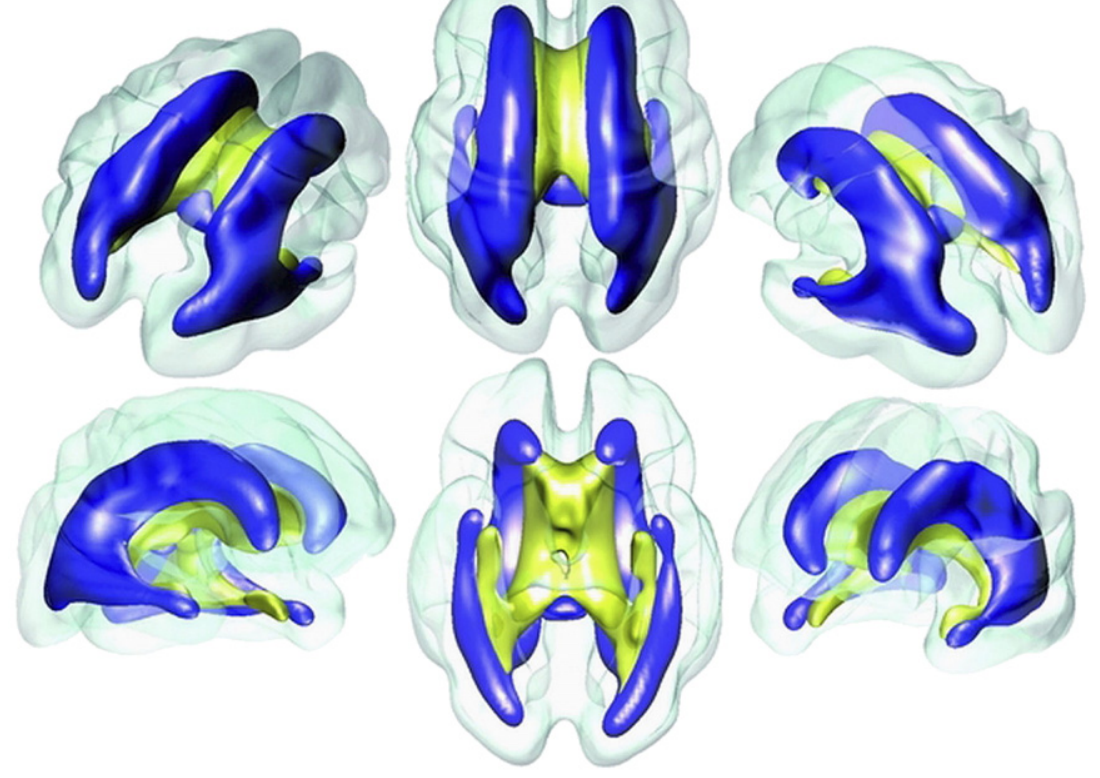

Fig. 1. Averaged lesion density map. Individual lesion density maps are created by blurring the automatically extracted binary lesion volumes with a Gaussian smoothing kernel. The 425 lesion density maps were averaged to create this averaged density map. This lesion density map (blue) follows the curvature of the lateral ventricles (yellow).

\section{Statistical analysis}

Age and sex were used as confounding covariates in each statistical model to control for their main effects on cortical thickness. Adding the acquisition site as a confounding covariate to the model did not change the results, therefore data are presented from the analysis without site as a covariate. Statistical analysis was performed at every vertex, regressing cortical thickness against TWMLL or disability scores. Although the EDSS is an ordinal scale, cortical thickness is normally distributed, therefore linear regressions were performed. In a separate analysis, we regressed cortical thickness against age, after removing the main effects of sex and total WM lesion volume. A final analysis correlated cortical thickness across the cortex with the lesion load measured separately in the anterior $(y>0)$ and posterior $(y<0)$ halves of the brain. This yielded two separate $t$-statistical maps that were subtracted (anterior minus posterior and vice-versa) in order to determine if there is a relationship between lesion location and local cortical thinning. The parameter of interest in these regression maps was the slope of the effect of TWMLL or disability score or age on cortical thickness. At each vertex, an estimate of the slope and its standard deviation were obtained by least squares, and $t$-statistic values were calculated by dividing the estimated slope by its estimated standard deviation. The resulting $t$-statistic map tested whether, at a given cortical vertex, the slope of the regression was significantly different from zero. The presence of significant peaks in these regression maps was assessed by a method based on random-field theory (Worsley et al., 1996, 1999), which corrects for multiple comparisons taking into account the search volume (entire cortex) and blurring. According to this method, a value of $t=4.4$ corresponds to $p=0.05$ (421 degrees of freedom, two-tailed, corrected for whole cortical search). We generated a standardized average brain surface to display the results of the statistical analysis by averaging the 425 cortices in stereotaxic space.

\section{Results}

The MS patients were mildly disabled, with a median EDSS score of 2 (range $0-8$ ). Only $12.7 \%$ had an EDSS score greater or equal to 4.5 at the time of the 24-month MRI exam. The mean relative TWMLL was $14.3 \mathrm{~cm}^{3}\left(\mathrm{SD}=14.3 \mathrm{~cm}^{3}\right.$, range $=0.2-$ $\left.78.6 \mathrm{~cm}^{3}\right)$.

\section{Regression of cortical thickness versus TWMLL}

Mean cortical thickness inversely correlated with the TWMLL (Fig. 3). The association was highly significant $(t=-15.3$, $p<0.0001$ ), representing an average loss of $0.03 \mathrm{~mm}$ in cortical thickness per cubic centimeter of WM lesion and accounting for $38 \%$ of the variance (as measured by the adjusted $R^{2}$ ). The regression of cortical thickness against TWMLL was statistically significant at almost every vertex of the surface model with few exceptions. Several cortical regions showed a more statistically significant association between the TWMLL and cortical thinning (Fig. 4 and Table 1) including bilateral cingulate gyrus, mostly in the anterior part (Brodmann's areas [BA] 32 and 24), and bilateral posterior insular cortex in the vicinity of the transverse temporal gyrus (BA 13 and 41). The association was also highly statistically significant bilaterally in prefrontal, temporal, and parietal association cortical regions whereas primary sensory, motor, and visual cortical regions showed a less statistically significant relationship. The highest degrees of focal cortical thinning per cubic centimeter of WM lesion tissue were found in the right $\left(0.07 \mathrm{~mm} / \mathrm{cm}^{3}\right)$ and left $\left(0.05 \mathrm{~mm} / \mathrm{cm}^{3}\right)$ anterior cingulate cortices (BA 24), as well as bilateral medial frontal gyrus (BA 6) $\left(0.05 \mathrm{~mm} / \mathrm{cm}^{3}\right)$. 


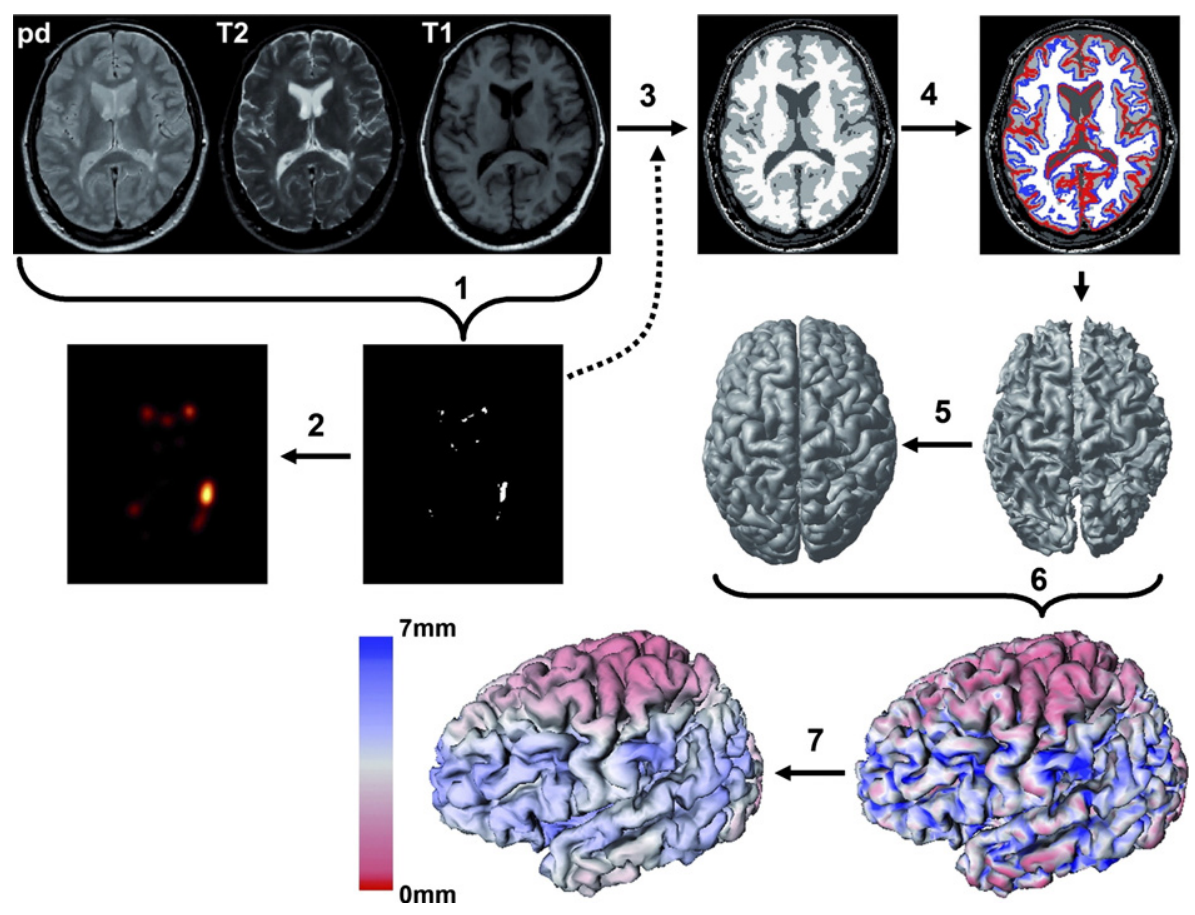

Fig. 2. Image processing steps. Native PD-, T2-, and T1-weighted MRIs enter the automated image processing pipeline (INSECT) for lesion extraction. The main steps of the pipeline are intensity non-uniformity correction, stereotaxic registration and resampling, and lesion extraction (1). The binary lesion volumes are smoothed with a Gaussian blurring kernel (full width at half maximum $=10 \mathrm{~mm}$ ) to create lesion 'density' maps (2). T1-weighted MRIs are classified with lesions masked out (3) and fit with a white matter surface (4). The gray surface is found by expanding out from the white (5). Cortical thickness is measured at every vertex (6), and blurred using a $20 \mathrm{~mm}$ surface-based kernel (7).

\section{Regression of cortical thickness versus EDSS scores}

Mean cortical thickness was also negatively correlated with the EDSS scores. The association was again highly significant ( $t=-4.1, p<0.0001$, adjusted $R_{2}=0.08$ ), representing an average loss of $0.08 \mathrm{~mm}$ in cortical thickness per EDSS unit. The most statistically significant correlations between EDSS scores and cortical thinning were found in bilateral middle and superior frontal gyri (BA 8, 9, and 10) as well as the anterior pole of the

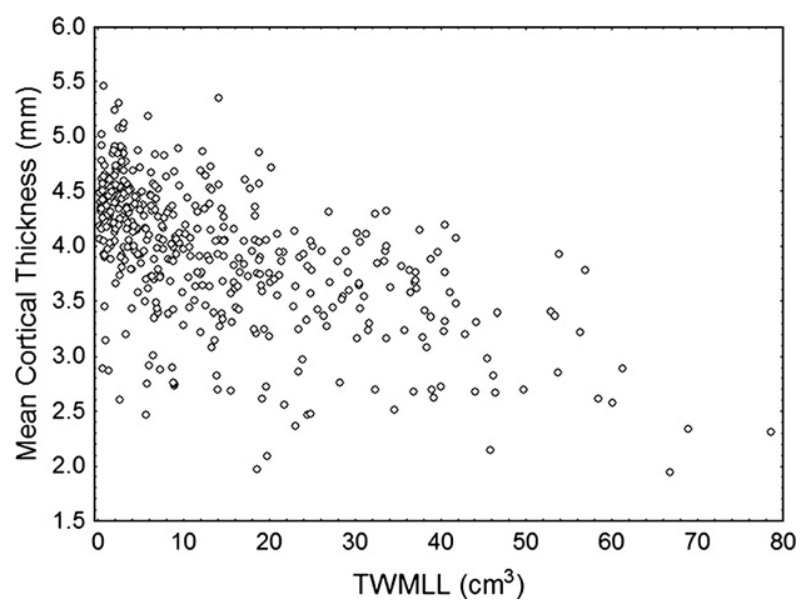

Fig. 3. Scatterplot showing the relationship between mean cortical thickness in millimeters and total white matter lesion load (TWMLL) in cubic centimeters in our MS 425 patients. left inferior temporal gyrus (BA 20). Other regions included bilateral anterior cingulate (BA 24) and left post-central cortical areas (Fig. 5 and Table 1). Similarly to the correlation between TWMLL and cortical thickness, the right $(0.24 \mathrm{~mm} / \mathrm{EDSS}$ unit $)$ and left $(0.21 \mathrm{~mm} / \mathrm{EDSS}$ unit) anterior cingulate cortices (BA 24) showed the highest degree of focal cortical thinning per EDSS unit. The left inferior temporal gyrus (BA 20) and inferior frontal gyrus (BA 47) had also a high amount of focal cortical thinning per EDSS unit $(0.20$ and $0.19 \mathrm{~mm} / \mathrm{EDSS}$ unit, respectively).

\section{Regression of cortical thickness versus age}

Mean cortical thickness negatively correlated with age $(t=-3$, $p<0.003$, adjusted $R^{2}=0.38$ ), representing an average loss of 0.1 $\mathrm{mm}$ in cortical thickness per decade. Regions where the association was most statistically significant included bilateral primary motor, somatosensory, and visual areas, as well as premotor cortex and inferior frontal gyrus.

Regression of cortical thickness versus WM lesion load in the anterior and posterior parts of the brain

In an attempt to show a relationship between lesion location and local cortical thinning, we generated two $t$-statistical maps by taking separately the WM lesion load in the anterior and posterior halves of the brain (defined as $y>0$ and $y<0$ ). Fig. 6 shows the resulting map that was obtained by subtracting the two $t$-statistical maps. In red are the regions were $t$-values were higher in the "anterior" $t$-statistical map than the "posterior" one. These red 

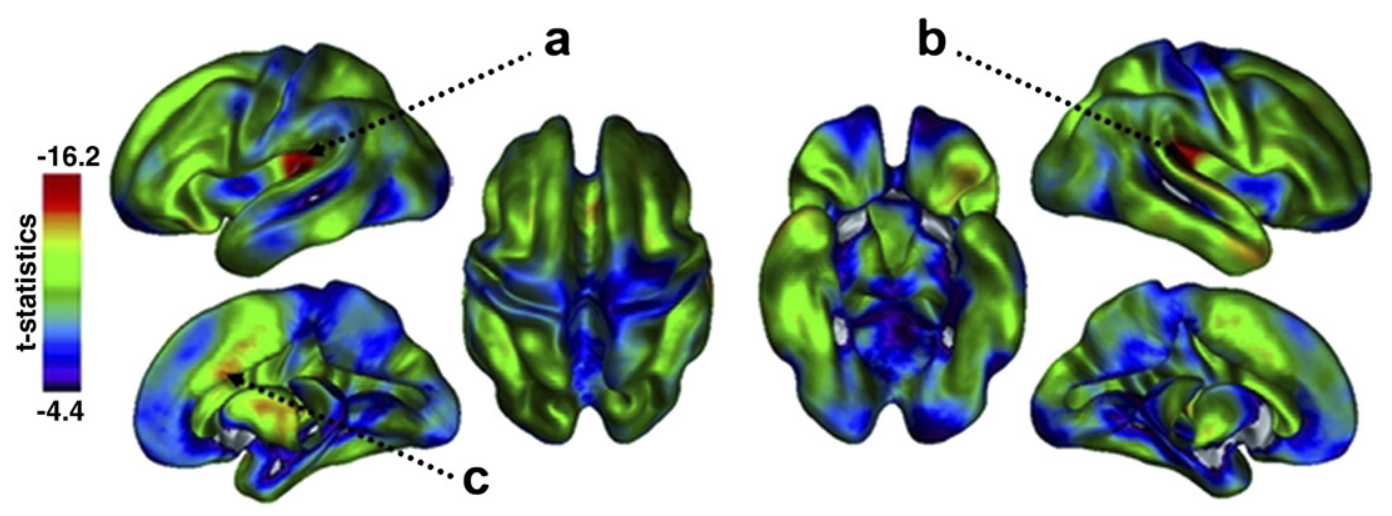

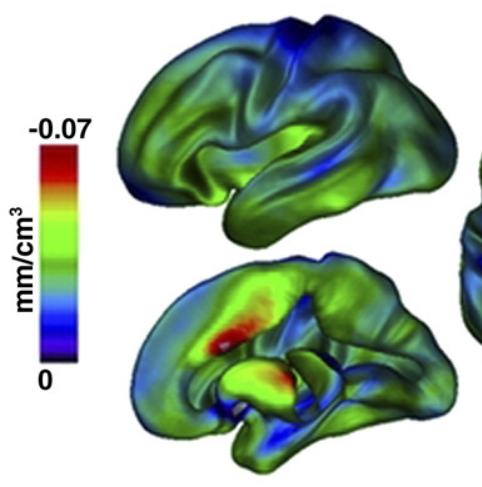

a

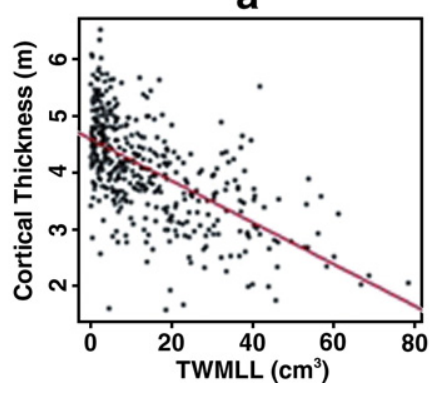

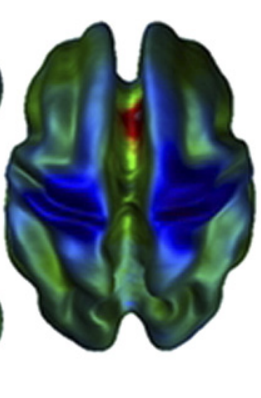

b

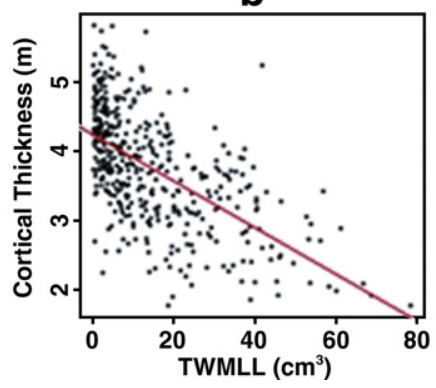

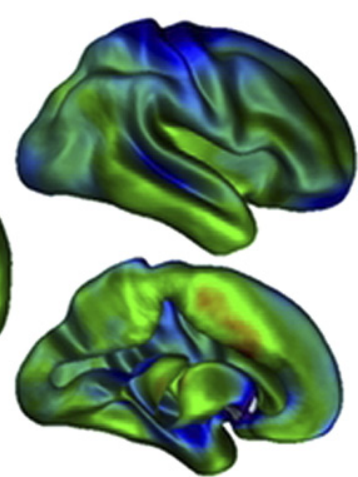

C

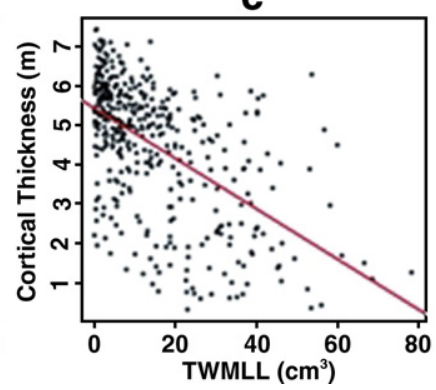

Fig. 4. $t$-statistical (top row) and cortical thickness (bottom row) maps of the TWMLL regression against cortical thickness in 425 MS subjects. Regions where cortical thickness correlates the most significantly with the TWMLL include bilateral anterior cingulate gyrus (c, right hemisphere), bilateral posterior insular cortex in the vicinity of the transverse temporal gyrus $(\mathrm{a}, \mathrm{b})$, right anterior middle temporal gyrus, and left inferior frontal and orbital gyri. The plots of cortical thickness against TWMLL are represented for locations a-c. TWMLL $=$ total white matter lesion load.

regions mostly include frontal and prefrontal cortices bilaterally. In blue are the regions were $t$-values were higher in the "posterior" $t$ statistical map the "anterior" one. The blue regions are mostly located in the inferior temporal and occipital regions.

\section{Discussion}

The fully automated method used in the present study has been previously applied to the study of brain development (Lerch et al., 2006; Shaw et al., 2006a, b) and Alzheimer's disease (Lerch et al., 2005), and now, for the first time, to 425 relapsingremitting MS patients in order to measure cortical thickness over the entire brain. We investigated the relation between both overall and focal cortical thickness and the TWMLL, and the EDSS.

We observed a significant negative correlation between mean cortical thickness and the total volume of WM lesions, confirming previous studies that used a volumetric approach
(Ge et al., 2001; Chard et al., 2002; De Stefano et al., 2003). We found that the correlation between the TWMLL and cortical thickness was statistically significant at almost every vertex of the surface model after correcting for multiple comparisons. The most statistically significant correlation was in bilateral cingulate gyrus, mostly in the anterior part and extending dorsally to reach the medial frontal gyrus (BA 6), and bilaterally in the posterior part of the insular cortex extending into the transverse temporal gyrus. These regions also displayed the greatest amount of cortical loss (in $\mathrm{mm}$ ) per unit of disability or lesion volume. The association was also highly significant in the left orbito-frontal and right anterior temporal cortices as well as bilaterally in prefrontal, temporal, and parietal cortical regions. The statistical significance of the relationship between atrophy and lesion load and the amount of cortical loss were lower in primary sensory, motor, and visual cortical regions. Disability was negatively correlated with the mean cortical thickness. The reduction in cortical thickness that correlated with the EDSS was especially 
Table 1

Localization of the areas where cortical thickness correlated most significantly with total white matter lesion load and disability scores

\begin{tabular}{|c|c|c|c|c|c|c|c|c|}
\hline & $x$ & $y$ & $z$ & $t$ & $\mathrm{~mm}$ loss & Location & & \\
\hline \multirow[t]{19}{*}{ TWMLL } & -59 & -40 & -21 & -12.0 & 0.04 & Left temporal lobe & Inferior temporal gyrus & BA 20,37 \\
\hline & -48 & 16 & 20 & -12.3 & 0.03 & Left frontal lobe & Inferior frontal gyrus & BA 9 \\
\hline & -39 & -51 & 42 & -12.3 & 0.03 & Left parietal lobe & Inferior parietal lobule & BA 40 \\
\hline & -37 & -26 & 10 & -16.2 & 0.04 & Left temporal lobe & Superior/Transverse temporal gyrus-posterior insular cortex & BA 13,41 \\
\hline & -35 & 28 & -17 & -13.5 & 0.03 & Left frontal lobe & Inferior/Orbital frontal gyrus & BA 47,11 \\
\hline & -26 & 12 & 52 & -12.8 & 0.03 & Left frontal lobe & Middle frontal gyrus & BA 6 \\
\hline & -20 & 45 & 35 & -11.7 & 0.03 & Left frontal lobe & Superior/Middle frontal gyrus & BA 8,9 \\
\hline & -8 & 20 & 32 & -12.7 & 0.05 & Left limbic lobe & Anterior cingulate gyrus & BA 32 \\
\hline & -7 & -4 & 52 & -12.5 & 0.05 & Left frontal lobe & Medial frontal gyrus & BA 6 \\
\hline & 7 & 20 & 24 & -14.1 & 0.07 & Right limbic lobe & Anterior cingulate gyrus & BA 24 \\
\hline & 8 & 0 & 49 & -12.2 & 0.05 & Right frontal lobe & Medial frontal gyrus & BA 6 \\
\hline & 24 & 38 & 39 & -12.8 & 0.03 & Right frontal lobe & Superior/Middle frontal gyrus & BA 8,9 \\
\hline & 29 & 2 & 53 & -12.8 & 0.03 & Right frontal lobe & Middle frontal gyrus & BA 6 \\
\hline & 34 & -47 & 46 & -11.7 & 0.03 & Right parietal lobe & Inferior parietal lobule & BA 40 \\
\hline & 44 & -24 & 9 & -16.2 & 0.04 & Right temporal lobe & Superior/Transverse temporal gyrus-posterior insular cortex & BA 13,41 \\
\hline & 51 & 8 & -32 & -13.7 & 0.04 & Right temporal lobe & Middle temporal gyrus & BA 21 \\
\hline & 52 & 26 & 15 & -12.5 & 0.03 & Right frontal lobe & Inferior frontal gyrus & BA 46 \\
\hline & 61 & -11 & 1 & -13.5 & 0.03 & Right temporal lobe & Superior temporal gyrus & BA 22 \\
\hline & 62 & -38 & -18 & -12.7 & 0.04 & Right temporal lobe & Inferior temporal gyrus & BA 20,37 \\
\hline \multirow[t]{9}{*}{ EDSS } & -35 & -3 & -40 & -5.7 & 0.20 & Left temporal lobe & Inferior temporal gyrus & BA 20 \\
\hline & -35 & 41 & 24 & -5.1 & 0.16 & Left frontal lobe & Middle frontal gyrus & BA 10 \\
\hline & -32 & -40 & 59 & -5.1 & 0.09 & Left parietal lobe & Postcentral gyrus & BA 5 \\
\hline & -24 & 45 & 28 & -5.0 & 0.14 & Left frontal lobe & Superior frontal gyrus & BA 9 \\
\hline & -17 & 16 & -20 & -5.5 & 0.19 & Left frontal lobe & Inferior frontal gyrus & BA 47 \\
\hline & -6 & 14 & 30 & -4.8 & 0.21 & Left frontal lobe & Anterior cingulate gyrus & BA 24 \\
\hline & 6 & 19 & 23 & -4.5 & 0.24 & Right frontal lobe & Anterior cingulate gyrus & BA 24 \\
\hline & 24 & 45 & 33 & -5.2 & 0.13 & Right frontal lobe & Superior frontal gyrus & BA 9 \\
\hline & 35 & 31 & 37 & -5.0 & 0.12 & Right frontal lobe & Middle frontal gyrus & BA 9 \\
\hline
\end{tabular}

$t$-values listed are statistically significant at $p<0.05$ corrected. Tissue loss is given in millimeters per cubic centimeter of white matter lesion or in millimeters per disability scale unit. BA=Brodmann's area; TWMLL=total white matter lesion load; EDSS=expanded disability status scale.

prominent in bilateral prefrontal and anterior cingulate regions, areas involved in many aspects of motor control (Jueptner et al., 1997).

For both correlations, the region that showed the highest amount of atrophy per scale unit was the anterior cingulate cortex. The anterior cingulate cortex has extensive cortico-cortical connections with other areas, including the lateral prefrontal, primary motor and premotor cortices, supplementary motor area, amygdala, striatum, thalamus, and brainstem (Paus, 2001). If cortical atrophy is the result of axonal transection by WM lesions, then highly connected regions should be more vulnerable than regions with relatively fewer connections. However, it is also possible that these gray matter regions are somehow intrinsically more vulnerable to direct involvement by the disease; indeed, histopathological studies have demonstrated that the cingulate gyrus, temporal lobe, and insula generally show a higher prevalence of cortical demyelinated lesions than other areas (Bö et al., 2003; Vercellino et al., 2005; Kutzelnigg and Lassmann, 2006).

Two studies on relatively small numbers ( $n=30$ and 26) of MS patients reported a correlation between fractional GM volume and total WM lesion volume, but not disability (Ge et al., 2001; Chard et al., 2002). However, another report in a larger patient group $(n=65)$ did uncover a correlation between GM volume and EDSS (De Stefano et al., 2003).

A recent longitudinal study (Chen et al., 2004), using a novel method to measure cortical thickness both globally and regionally, compared the rate of cortical atrophy in two groups of MS patients based on whether they had a stable $(n=20)$ or progressing $(n=10)$ disability over a one year period. The group whose disability progressed over the 1 year inter-scan interval showed a higher average rate of cortical GM thinning than the group that remained stable, suggesting that disability progression and rates of global cortical atrophy are related.

Sailer and colleagues (2003) showed that 20 patients with clinically definite MS had significant cortical atrophy compared to controls, and that the degree of atrophy correlated with the disability score and lesion volume. Focally, thinning of the cortex was found bilaterally in frontal and temporal association areas, even in patients with short disease durations or mild impairment. However, only subjects with severe disability (EDSS $\geq 6$ ), or with higher total lesion volume, showed additional small areas of focal cortical atrophy in the vicinity of the premotor and motor cortices. Overall, our results are very similar. We did not find significant correlations between cortical thinning and EDSS scores in the premotor and motor cortices, in our relatively mildly impaired patient group.

The pattern of cortical atrophy we describe is distinctly different from that seen with normal aging. Measurements of cortical thickness in healthy subjects show age-related atrophy mostly in primary motor and premotor cortices, calcarine cortex near the primary visual cortex, and prefrontal regions (Salat et al., 2004). We found that regions where the relationship between the TWMLL and cortical loss was most significant included the anterior cingulate cortex, the insula and transverse temporal gyrus, and several areas in association cortex. In clear distinction to the 

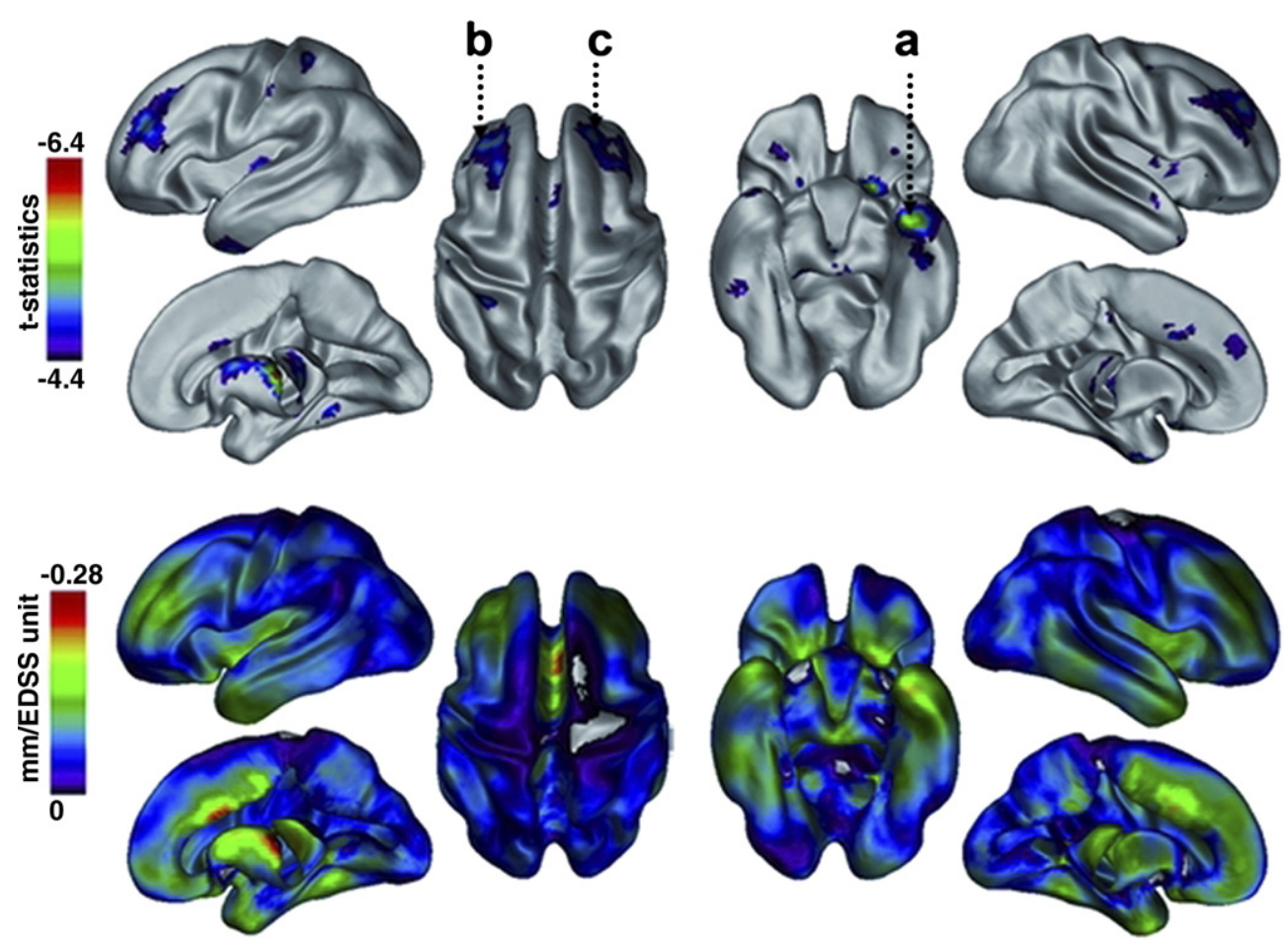

a

b
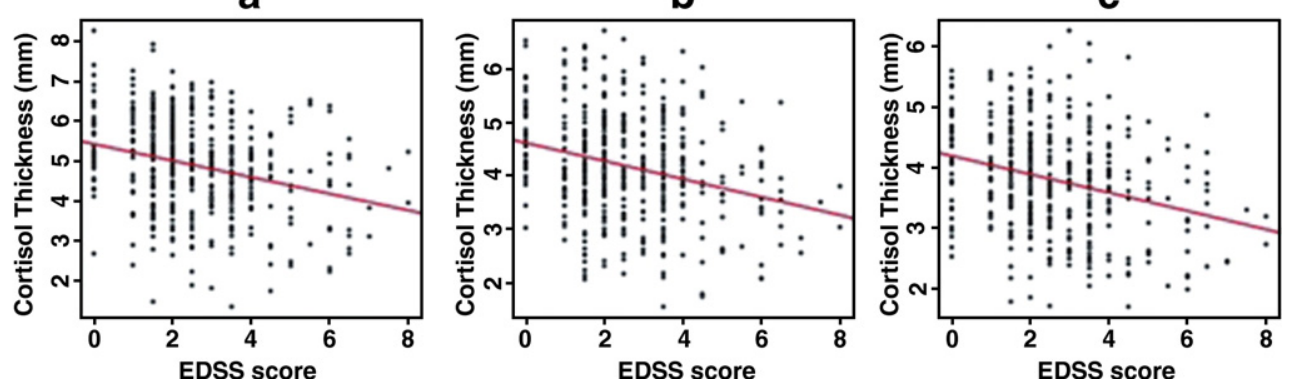

Fig. 5. $t$-statistical (top row) and cortical thickness (bottom row) maps of the EDSS regression against cortical thickness in 425 MS subjects. Regions where cortical thickness correlates the most significantly with the EDSS include the middle and superior frontal gyri bilaterally (b, c), the left inferior temporal gyrus (a), as well as bilateral anterior cingulate cortex. The plots of cortical thickness against TWMLL are represented for locations a-c. EDSS=expanded disability status scale.

results reported in normal aging (Salat et al., 2004), the correlation was weakest in primary sensory, motor, and visual cortices. The anterior cingulate cortex, the region where the amount of thinning per cubic centimeter of WM lesion was the highest in our study, was reported by Salat and colleagues (2004) as a region that tends to get thicker with age. Thus, our data suggest that cortical morphological changes in MS follow a path that is specific to the disease. It is noteworthy that the correlation between cortical thickness and age in our group yielded results very similar to those seen in studies in healthy controls, which serves to validate our methodology.

The location of WM lesions could affect the pattern of focal atrophy. This is in part supported by our findings of a relationship between the WMLL in the anterior and posterior parts of the brain and preferential thinning of the cortex accordingly in anterior and posterior regions (Fig. 6). The averaged lesion density map (Fig. 1) shows that lesion density is particularly high in periventricular $\mathrm{WM}$, and lesions there would interrupt tracts that originate from or project to prefrontal, cingulate, and association areas. This could help explain why heavily connected cortical areas, such as anterior cingulate, insula, and association cortex, might be more vulnerable to atrophy.

It is perhaps surprising to observe a limited atrophy of primary motor and visual areas since MS lesions are often located in the cortico-spinal tracts and optic nerves. One possibility is that the method used here to measure cortical thickness is less sensitive to variations in these regions. However, this is unlikely since the correlation between cortical thickness and age, in our patient sample, revealed statistically significant areas bilaterally in premotor and primary sensorimotor and visual cortices, confirming previous findings in normal aging (Salat et al., 2004). The low degree of cortical thinning per either TWMLL or disability score in primary sensorimotor areas in our relatively mildly impaired patients suggests that atrophy of primary motor cortex preferentially appears later in the disease course (Sailer et al., 2003).

There are limitations to our approach. One is the inability to detect inflammatory GM lesions with the conventional imaging 


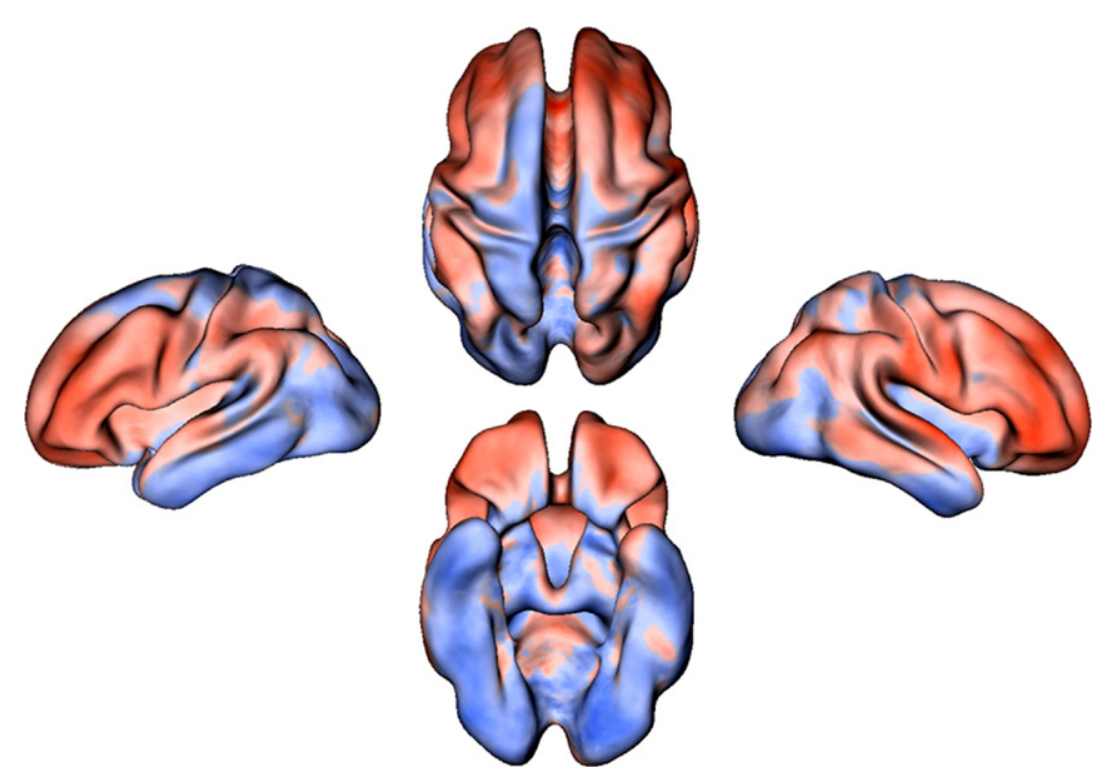

Fig. 6. Relationship between regional lesion load and local cortical thickness. White matter lesion loads were measured separately in the anterior $(y>0)$ and posterior $(y<0)$ parts of the brain and correlated with cortical thickness across the entire cortex. In red are the regions were $t$-values were higher in the "anterior" $t$-statistical map than the "posterior" one. These red regions mostly include frontal and prefrontal cortices bilaterally. In blue are the regions were $t$-values were higher in the "posterior" $t$-statistical map the "anterior" one. The blue regions are mostly located in the inferior temporal and occipital regions.

techniques used in our protocol. Future cortical atrophy studies in MS could benefit from the use of MRI sequences developed to better detect superficial brain lesions (Geurts et al., 2005). We cannot rule out the possibility of cortical demyelinating lesions interfering with the cortical thickness measurements.

Another limitation was the use of non-isotropic voxels in this clinical trial. The relatively low spatial resolution in the $z$ direction ( $3 \mathrm{~mm}$ slice thickness) of the T1-weighted scans could limit the sensitivity of the cortical thickness measurement; however, this is balanced by the high number of subjects $(n=425)$. Second, voxel anisotropy could potentially lead to a bias in the measurement of cortical atrophy, making our method more sensitive to change in certain cortical areas relative to others. However, the finding of age-correlated cortical thickness in primary sensory, motor, and visual areas in this data set confirms that the method is not insensitive to atrophy in these areas. Thus our finding of MS-related atrophy in predominantly associative rather then primary areas cannot be explained by a methodological bias.

Juxta-cortical WM lesions are known to occur in MS and could interfere with the cortical thickness measurement. We attempted to overcome this limitation by masking out juxta-cortical WM lesions prior to the determination of the WM and GM contours (by labeling these lesions as WM for this purpose). Of course, we cannot exclude the possibility that juxta-cortical lesions undetected by the automatic segmentation could have introduced errors into our cortical thickness estimates.

This is the first large-scale study of focal cortical atrophy in MS that uses cortical thickness measurements across the entire cortex. As opposed to methods that provide a general measure of cortical atrophy, such as normalized cortical volumes, our method measures cortical thickness at each vertex across the entire brain. This allows statistical analysis on a vertex by vertex basis revealing focal areas of cortical atrophy correlating with specific measures of disability or lesion burden. Our data suggest that cortical atrophy occurs even in mildly disabled patients, that it is progressive, and that it follows a pattern that is distinctively different from the one in normal aging. Further studies are required to investigate, more precisely, the relation between WM lesion location and the pattern of focal cortical atrophy. Finally, more specific evaluation of different clinical functions, such as motor and cognitive impairment (Cutter et al., 1999), depression, or fatigue, could increase the sensitivity of correlation analyses.

\section{Acknowledgments}

The MRI and clinical database was collected as part of a phase III clinical trial of oral myelin (Myloral) for treatment of multiple sclerosis, financed by Autoimmune Inc. of Boston, MA. The analysis reported in this study has been supported in part by NIH Human Brain Project award P01MH52176-06 to the International Consortium for Brain Mapping (ICBM) and by Canadian Institutes of Health Research Grants MOP 37754, MOP 14780, and MGC 13792 awarded to Evans and Dagher. Dr. Dagher is supported by the Fonds de Recherche en Santé du Québec, and the Canadian Institute for Health Research. A. Charil was funded by the Multiple Sclerosis Society of Canada.

We also acknowledge the participating sites in the imaging component of the Myloral study: St. Michael's Hospital (Toronto, ON), Montreal Neurological Hospital (Montreal, PQ), University of British Colombia (Vancouver, BC), Ottawa General Hospital (Ottawa, ON), University of Maryland (Baltimore, MD), Lehigh Magnetic Imaging Center (Allentown, PA), Maimonides Medical Center Brooklyn, NY), St. John Medical Center (Tulsa, OK), University of Minnesota (Minneapolis, MN), Washington University (St. Louis, MO), Cornell Medical Center (New York, NY), University of Southern California (Los Angeles, CA), and Carolinas Medical Center (Charlotte, NC). 


\section{References}

Anderson, V.M., Fox, N.C., Miller, D.H., 2006. Magnetic resonance imaging measures of brain atrophy in multiple sclerosis. J. Magn. Reson. Imaging 23, 605-618.

Ashburner, J., Friston, K.J., 2001. Voxel-based morphometry-The methods. NeuroImage 11, 805-821.

Bakshi, R., Benedict, R.H., Bermel, R.A., Jacobs, L., 2001. Regional brain atrophy is associated with physical disability in multiple sclerosis: semiquantitative magnetic resonance imaging and relationship to clinical findings. J. Neuroimaging 11, 129-136.

Bö, L., Vedeler, C.A., Nyland, H.I., Trapp, B.D., Mork, S.J., 2003. Subpial demyelination in the cerebral cortex of multiple sclerosis patients. J. Neuropathol. Exp. Neurol. 62, 723-732.

Chard, D.T., Griffin, C.M., Parker, G.J., Kapoor, R., Thompson, A.J., Miller, D.H., 2002. Brain atrophy in clinically early relapsing-remitting multiple sclerosis. Brain 125, 327-337.

Charil, A., Zijdenbos, A.P., Taylor, J., Boelman, C., Worsley, K.J., Evans, A.C., Dagher, A., 2003. Statistical mapping analysis of lesion location and neurological disability in multiple sclerosis: application to 452 patient data sets. NeuroImage 19, 532-544.

Chen, J.T., Narayanan, S., Collins, D.L., Smith, S.M., Matthews, P.M., Arnold, D.L., 2004. Relating neocortical pathology to disability progression in multiple sclerosis using MRI. NeuroImage 23, 1168-1175.

Chung, M.K., Worsley, K.J., Robbins, S., Paus, T., Taylor, J., Giedd, J.N., Rapoport, J.L., Evans, A.C., 2003. Deformation-based surface morphometry applied to gray matter deformation. NeuroImage 18, 198-213.

Collins, D.L., Neelin, P., Peters, T.M., Evans, A.C., 1994. Automatic 3D intersubject registration of MR volumetric data in standardized Talairach space. J. Comput. Assist. Tomogr. 18, 192-205.

Cutter, G.R., Baier, M.L., Rudick, R.A., Cookfair, D.L., Fischer, J.S., Petkau, J., Syndulko, K., Weinshenker, B.G., Antel, J.P., Confavreux, C., Ellison, G.W., Lublin, F., Miller, A.E., Rao, S.M., Reingold, S., Thompson, A., Willoughby, E., 1999. Development of a multiple sclerosis functional composite as a clinical trial outcome measure. Brain $122,871-882$.

De Stefano, N., Matthews, P.M., Filippi, M., Agosta, F., De Luca, M., Bartolozzi, M.L., Guidi, L., Ghezzi, A., Montanari, E., Cifelli, A., Federico, A., Smith, S.M., 2003. Evidence of early cortical atrophy in MS: relevance to white matter changes and disability. Neurology 60 , $1157-1162$.

Eastwood, A., Reingold, S., 1997. Disappointing results from clinical trial of oral myelin for relapsing-remitting multiple sclerosis. Research and Medical Programs Department News. National Multiple Sclerosis Society, New York, NY.

Ge, Y., Grossman, R.I., Udupa, J.K., Babb, J.S., Nyul, L.G., Kolson, D.L., 2001. Brain atrophy in relapsing-remitting multiple sclerosis: fractional volumetric analysis of gray matter and white matter. Radiology 220 , 606-610.

Geurts, J.J., Pouwels, P.J., Uitdehaag, B.M., Polman, C.H., Barkhof, F., Castelijns, J.A., 2005. Intracortical lesions in multiple sclerosis: improved detection with 3D double inversion-recovery MR imaging. Radiology 236, 254-260.

Hardmeier, M., Wagenpfeil, S., Freitag, P., Fisher, E., Rudick, R.A., KooijmansCoutinho, M., Clanet, M., Radue, E.W., Kappos, L., European rIFN beta-1a in Relapsing MS Dose Comparison Trial Study Group, 2003. Atrophy is detectable within a 3-month period in untreated patients with active relapsing remitting multiple sclerosis. Arch. Neurol. 60, 1736-1739.

Jueptner, M., Stephan, K.M., Frith, C.D., Brooks, D.J., Frackowiak, R.S., Passingham, R.E., 1997. Anatomy of motor learning: I. Frontal cortex and attention to action. J. Neurophysiol. 77, 1313-1324.

Kabani, N., Le Goualher, G., MacDonald, D., Evans, A.C., 2001. Measurement of cortical thickness using an automated 3-D algorithm: a validation study. NeuroImage 13, 375-380.

Kalkers, N.F., Ameziane, N., Bot, J.C., Minneboo, A., Polman, C.H., Barkhof, F., 2002. Longitudinal brain volume measurement in multiple sclerosis: rate of brain atrophy is independent of the disease subtype. Arch. Neurol. 59, 1572-1576.

Kamber, M., Shinghal, R., Collins, L., Francis, G.S., Evans, A.C., 1995. Model-based 3D segmentation of multiple sclerosis lesions in magnetic resonance brain images. IEEE Trans. Med. Imag. 14, 442-453.

Kim, J.S., Singh, V., Lee, J.K., Lerch, J., Ad-Dab'bagh, Y., MacDonald, D., Lee, J.M., Kim, S.I., Evans, A.C., 2005. Automated 3-D extraction and evaluation of the inner and outer cortical surfaces using a Laplacian map and partial volume effect classification. NeuroImage 27, 210-221.

Kurtzke, J.F., 1983. Rating neurologic impairment in multiple sclerosis: an expanded disability status scale (EDSS). Neurology 33, 1444-1452.

Kutzelnigg, A., Lassmann, H., 2006. Cortical demyelination in multiple sclerosis: a substrate for cognitive deficits? J. Neurol. Sci. 245, 123-126.

Lerch, J.P., Evans, A.C., 2005. Cortical thickness analysis examined through power analysis and a population simulation. NeuroImage 24, 163-173.

Lerch, J.P., Pruessner, J.C., Zijdenbos, A., Hampel, H., Teipel, S.J., Evans, A.C., 2005. Focal decline of cortical thickness in Alzheimer's disease identified by computational neuroanatomy. Cereb. Cortex 15, 995-1001.

Lerch, J.P., Worsley, K., Shaw, W.P., Greenstein, D.K., Lenroot, R.K., Giedd, J., Evans, A.C., 2006. Mapping anatomical correlations across cerebral cortex (MACACC) using cortical thickness from MRI. NeuroImage 31, 993-1003.

Paus, T., 2001. Primate anterior cingulate cortex: where motor control, drive and cognition interface. Nat. Rev., Neurosci. 2, 417-424.

Peterson, J.W., Bo, L., Mork, S., Chang, A., Trapp, B.D., 2001. Transected neurites, apoptotic neurons, and reduced inflammation in cortical multiple sclerosis lesions. Ann. Neurol. 50, 389-400.

Poser, C.M., Paty, D.W., Scheinberg, L., McDonald, W.I., Davis, F.A., Ebers, G.C., Johnson, K.P., Sibley, W.A., Silberberg, D.H., Tourtellotte, W.W., 1983. New diagnostic criteria for multiple sclerosis: guidelines for research protocols. Ann. Neurol. 13, 227-231.

Sailer, M., Fischl, B., Salat, D., Tempelmann, C., Schonfeld, M.A., Busa, E., Bodammer, N., Heinze, H.J., Dale, A., 2003. Focal thinning of the cerebral cortex in multiple sclerosis. Brain 126, 1734-1744.

Salat, D.H., Buckner, R.L., Snyder, A.Z., Greve, D.N., Desikan, R.S., Busa, E., Morris, J.C., Dale, A.M., Fischl, B., 2004. Thinning of the cerebral cortex in aging. Cereb. Cortex 14, 721-730.

Shaw, P., Lerch, J., Clasen, L., Lenroot, R., Gogtay, N., Evans, A., Rapoport, J., Giedd, J., 2006a. Intellectual ability and cortical development in children and adolescents. Nature 440, 676-679.

Shaw, P., Greenstein, D., Lerch, J., Sharp, W., Clasen, L., Evans, A., Giedd, J., Castellanos, F.X., Rapoport, J., 2006b. Longitudinal mapping of cortical thickness and clinical outcome in children and adolescents with attention deficit/hyperactivity disorder. Arch. Gen. Psychiatry 63, $540-549$.

Sled, J.G., Zijdenbos, A.P., Evans, A.C., 1998. A nonparametric method for automatic correction of intensity nonuniformity in MRI data. IEEE Trans. Med. Imag. 17, 87-97.

Talairach, J., Tournoux, P., 1988. Co-planar Stereotaxic Atlas of the Human Brain: 3-Dimensional Proportional System: An Approach to Cerebral Imaging. Thieme, Stuttgart.

Vercellino, M., Plano, F., Votta, B., Mutani, R., Giordana, M.T., Cavalla, P., 2005. Grey matter pathology in multiple sclerosis. J. Neuropathol. Exp. Neurol. 64, 1101-1117.

Weiner, H.L., 1997. Oral tolerance for the treatment of autoimmune diseases. Annu. Rev. Med. 48, 341-351.

Worsley, K.J., Marrett, S., Neelin, P., Vandal, A.C., Friston, K.J., Evans, A.C., 1996. A unified statistical approach for determining significant signals in images of cerebral activation. Hum. Brain Mapp. 4, $58-73$.

Worsley, K.J., Andermann, M., Koulis, T., MacDonald, D., Evans, A.C., 1999. Detecting changes in nonisotropic images. Hum. Brain Mapp. 8, 98-101.

Zijdenbos, A.P., Forghani, R., Evans, A.C., 2002. Automatic "pipeline" analysis of 3-D MRI data for clinical trials: application to multiple sclerosis. IEEE Trans. Med. Imag. 21, 1280-1291. 